Western University

Scholarship@Western

Brain and Mind Institute Researchers'

Publications

Brain and Mind Institute

3-1-2016

Category learning in older adulthood: A study of the Shepard, Hovland, and Jenkins (1961) tasks.

Rahel Rabi

Department of Psychology, The University of Western Ontario

John Paul Minda

Department of Psychology, The University of Western Ontario

Follow this and additional works at: https://ir.lib.uwo.ca/brainpub

Part of the Neurosciences Commons, and the Psychology Commons

Citation of this paper:

Rabi, Rahel and Minda, John Paul, "Category learning in older adulthood: A study of the Shepard, Hovland, and Jenkins (1961) tasks." (2016). Brain and Mind Institute Researchers' Publications. 295.

https://ir.lib.uwo.ca/brainpub/295 


\title{
Category Learning in Older Adulthood: A Study of the Shepard, Hovland, and Jenkins (1961) Tasks
}

\author{
Rahel Rabi and John Paul Minda \\ The University of Western Ontario
}

\begin{abstract}
Shepard, Hovland, and Jenkins (1961) examined the categorization abilities of younger adults using tasks involving single-dimensional rule learning, disjunctive rule learning, and family resemblance learning. The current study examined category learning in older adults using this well-known category set. Older adults, like younger adults, found category tasks with a single relevant dimension the easiest to learn. In contrast to younger adults, older adults found complex disjunctive rule-based categories harder to learn than family resemblance based categories. Disjunctive rule-based category learning appeared to be the most difficult for older adults to learn because this category set placed the heaviest demands on working memory, which is known to be a cognitive function that declines with normal aging. The authors discuss why complex rule-based category learning is considered more difficult for older adults to learn relative to younger adults, drawing parallels to developmental research.
\end{abstract}

Keywords: category learning, rules, working memory, inhibitory control, aging

The ability to categorize is a key aspect of cognition, which we rely on to group like objects together so that they can later be treated equivalently. Starting from infancy and continuing to older adulthood, we make categorization decisions to help organize the world around us. That is, children may rely on categorization when deciding whether some types of objects are dangerously hot (e.g., stove) or not (e.g., fridge), whereas older adults might rely on categorization to decide which types of medications are dangerous or safe. This being said, a principal question to examine is whether category learning abilities vary with age, and which factors are responsible for these changes. Developmental differences in category learning have been examined in early and middle childhood (Minda, Desroches, \& Church, 2008; Huang-Pollock, Maddox, \& Karalunas, 2011; Rabi \& Minda, 2014), and category learning abilities have been extensively studied in university-aged adults. At the opposite end of the age spectrum, category learning abilities in older adulthood have only recently begun to receive attention. Given the fact that quick and accurate categorization is just as important later in life as it is earlier in life, it is important to examine if and why age-related changes in category learning may appear in older adulthood.

This article was published Online First January 14, 2016.

Rahel Rabi and John Paul Minda, Department of Psychology, The University of Western Ontario.

This research was supported by an NSERC (The Natural Sciences and Engineering Research Council of Canada) and SSHRC (The Social Sciences and Humanities Research Council of Canada) grant to John Paul Minda.

Correspondence concerning this article should be addressed to Rahel Rabi, Department of Psychology, The University of Western Ontario, London, Ontario N6A 5C2, Canada. E-mail: rrabi@uwo.ca

\section{Parallels Between Category Learning in Childhood and Older Adulthood}

Given the limited research on category learning in older adults, a better understanding of this topic may be obtained by examining category learning in children. On the surface, it may appear as though these two populations have little in common. In addition, there are also vast differences in semantic knowledge between these two groups which prompt some caution in making comparisons and inferences between them. Despite these differences, these groups do share some key similarities that can inform theorization. For example, research suggests that the prefrontal cortex develops later than other areas (Bunge \& Zelazo, 2006; Kolb et al., 2012), and verbal working memory and executive functioning develop substantially during childhood and are related to these physical developments in the prefrontal cortex (Gathercole, 1999). As a result, children are often impaired relative to younger adults when learning categories that rely heavily on working memory and executive functioning abilities (Minda et al., 2008; Rabi \& Minda, 2014). Similarly, research has shown that prefrontal brain regions atrophy with normal aging, which is associated with a reduction in executive functioning abilities (Greenwood, 2000; Grieve, Williams, Paul, Clark, \& Gordon, 2007). Furthermore, one might expect that older adults should also be impaired when learning category sets that depend on working memory and executive functioning.

Research by Minda and colleagues (2008) showed that children performed worse than younger adults on categories that were optimally learned by a complicated rule. However, children and younger adults performed similarly when learning nonrule-based family resemblance (FR) categories, which could be learned based on the overall similarity of the stimulus dimensions rather than via a rule. Interestingly, children were able to learn simple, singledimensional rules about as well as younger adults, suggesting that children are capable of learning rules if they are easy to identify (i.e., do not heavily tax working memory and inhibitory control 
abilities). Along the same lines, Huang-Pollock et al. (2011) found that adults outperformed children on rule-based (RB) categories because children overly relied on suboptimal single dimensional rules when solving both category sets. Recent research by Rabi and Minda (2014) extended the results of Huang-Pollock et al. by showing that not only are children impaired at RB category learning compared to adults, but that these impairments are also related to their executive functioning abilities. That is, Rabi and Minda demonstrated that working memory and inhibitory control are associated with RB category learning, and as these abilities improved with age, so did RB category learning performance. Furthermore, given that prefrontal functioning changes with age and there are observed reductions in executive functioning abilities (Raz, 2000; Braver et al., 2001) it is reasonable to expect to see larger RB deficits in older adults compared to nonrule-based FR deficits.

\section{Rule-Use in Older Adults}

Aside from developmental research on category learning, examining general rule-learning abilities in older adults can help shed light on how and why category learning abilities change with age. The Wisconsin Card Sorting Test (WCST) has frequently been used to assess rule learning. In this task, participants learn to categorize multidimensional stimuli based on a single-dimensional rule (e.g., shape), using feedback to determine when to switch rules. Various studies have revealed that WCST performance tends to decline with age (Hayslip \& Sterns, 1979; Axelrod \& Henry, 1992; but see Gorlick et al., 2013 for an exception). More specifically, Ridderinkhof, Span, \& van der Molen (2002) found that decrements in WCST performance could be attributed to the fact that older adults perseverated on previously correct sorting rules, even when provided with explicit cues (e.g., "shift to color") to aid learning. These findings suggest that older adults may struggle to use rules appropriately because of difficulties with hypothesis testing and set-shifting abilities. Along the same lines, Chasseigne, Mullet, and Stewart (1997) examined the effects of aging on multiple cue probability learning (i.e., learning the probabilistic relationship between cues and events) in older adults (65-75 and 76-90 years old) and younger adults. When there was a direct relationship between cues and events, all participants performed similarly. However, when the cue and event were inversely related to each other, younger adults outperformed older adults. Interestingly, in a second task where participants were explicitly told about the inverse relationship, 65- to 75-year-olds showed improved performance, but 76- to 90-year-olds continued to show impairments. Furthermore, these findings suggest that older adults may find it difficult to use explicit rules in specific situations, possibly due to declining working memory abilities. That is, arriving at the inverse relationship would involve creating a verbal rule, which could be quite taxing on working memory.

\section{Category Learning in Older Adults}

While studies have clearly shown an age-related reduction in the propensity to use rules, research has also demonstrated more specific category learning deficits in older adulthood. For example, research conducted by Filoteo and Maddox (2004) examined the ability of younger adults and older adults to learn information- integration categories, which are a subset of nonrule-based categories. One category set was defined by a linear boundary, thus making it comparable to a family resemblance category set, and the other was defined by a non linear boundary. In both cases, younger adults performed better than the older adults. One reason was that younger adults were more adept at switching to an information integration strategy whereas older adults were more likely to use a rule-based approach (which resulted in suboptimal performance).

Other research examined rule-based category learning. Racine, Barch, Braver, and Noelle (2006) asked older adults (ages 66 to 82) and younger adults to learn a set of categorization tasks that varied in rule complexity. Results revealed that older adults performed similarly to younger adults when applying a simple, singledimensional rule, but showed performance deficits when applying a more complicated rule. Racine's findings converge nicely with Minda et al.'s (2008) developmental results. Similar to Racine and colleagues, Minda et al. (2008) also found that children struggled with learning complex rules, but performed similarly to younger adults when learning simpler, single-dimensional categorization rules. Maddox, Pacheco, Reeves, Zhu, and Schnyer (2010), also examined RB category learning in older adults (ages 60 to 81), paying special attention to strategy use differences in younger and older adults. Findings revealed that as a group, older adults showed RB deficits compared to younger adults. Computational modeling provided further insight into the types of strategies being used by older adults, revealing that older adults were marginally less likely to use the task appropriate strategy in the RB condition compared to younger adults. Among the older adults who did not use an explicit, hypothesis-testing strategy, these participants tended to rely on either a nonrule-based implicit strategy or guessing. Maddox et al. (2010) also showed that older adults who adopted the task appropriate strategy (i.e., a hypothesis-testing strategy) in the $\mathrm{RB}$ condition performed similarly to younger adults using the task appropriate strategy. Additionally, Maddox et al. demonstrated that older adults who used the task appropriate strategy were also those who showed better inhibitory control (on the WCST and Stroop task) and working memory (on the digit span task) abilities. These findings are clearly in line with research showing the executive functioning abilities are closely tied to RB category learning (Miles \& Minda, 2011; Minda et al., 2008) and tend to decline with age (Greenwood, 2000; Raz, 2000).

Another type of category learning that has been examined in older adults is rule and exception learning. Davis, Love, and Maddox (2012) asked older and younger adults to learn to categorize pictures of beetles on the basis of trial and error. Most of the beetles were rule-following items that could be categorized using a single dimensional rule. However, each category also contained an exception item. Davis and colleagues found that while both older and younger adults performed quite well on the rulefollowing items by the end of training, older adults were impaired at categorizing the exception items. In line with the findings of Racine and colleagues (2006), Davis et al. demonstrated that older adults could learn RB category sets, granted that the verbal rule was straightforward (e.g., beetles with pointy antenna go in Category A). However, older adults struggled more than younger adults, when they had to exert additional resources (e.g., hypothesis testing, working memory) to determine the exception to the rule. 


\section{Changes in Executive Functioning With Age}

Given the limited number of studies investigating category learning in older adults, a useful next step would be to outline some of the factors which may impact category learning in older adults. One of the factors that are known to influence category learning is executive functioning. According to widely held views, the prefrontal cortex plays a key role in executive functioning. However, this brain region has also been shown to deteriorate with age (van der Mollen \& Ridderinkhof, 1998; Uylings \& de Brabander, 2002). Future research should closely examine the link between category learning deficits in older adults and executive functioning abilities, because many types of category learning tasks depend on executive functioning abilities like inhibitory control and working memory (Miyake et al., 2000).

\section{Inhibitory Control}

Older adults have been shown to display deficits in a wide range of inhibition tasks. For example, prior research has shown that older adults find it more difficult to look away from an onset stimulus when the correct response is to look in the opposite direction (Butler, Zacks, \& Henderson, 1999; Olincy, Ross, Youngd, \& Freedman, 1997). Additionally, when older adults are required to stop their response when a target stimulus is presented (i.e., stop-signal task), they have more difficulty withholding their response than younger adults (May \& Hasher, 1998; Williams, Ponesse, Schachar, Logan, \& Tannock, 1999). With regards to the Stroop task, research has shown that older adults find it more difficult to suppress the word reading response to a color word when asked to indicate the font color of the word (West, 1999; Davidson, Zacks, \& Williams, 2003). Furthermore, these findings demonstrate that inhibitory processes are impaired in older adults, which may have an impact on how well older adults can learn categories. That is, reduced inhibitory control abilities may lead to more difficulty restricting access of irrelevant/salient information to working memory, as well as difficulty removing information from working memory that has been deemed irrelevant.

\section{Working Memory}

Age related decrements in working memory performance have also been documented. Research has revealed decreases in work- ing memory in old age in both verbal and spatial working memory tasks (Bopp \& Verhaeghen, 2005; Park et al., 2002). With regards to category learning, Lewandowsky (2011) found that working memory capacity mediated performance on RB tasks. As well, Maddox et al. (2010) found that digit span performance was associated with RB category learning in older adults. Based on prior research, we know that older adults are capable of learning simple, single-dimensional RB categories, but struggle to learn more complex RB categories (Racine et al., 2006). One might speculate that older adults struggled to learn the more complicated RB category set, because it placed more strain on their working memory capacity to test different rules, update the information, and maintain the complex rule in memory.

\section{Motivation for the Current Research}

The current study examined category learning in younger and older adults using an adapted version of the Shepard, Hovland, and Jenkins' (1961) classification tasks (hereafter referred to as the SHJ tasks). This category set has been used in many studies across different population types (e.g., younger adults, children, depressed individuals, monkeys), but to our knowledge, it has yet to be examined in older adults. Because it has been used so extensively, performance on the SHJ tasks serves as an important benchmark for understanding category learning. It follows that performance on these category sets should be examined in relation to normal aging. Among the six SHJ category sets, three (Type I, Type II, and Type IV) were of particular interest in the present study. Type I is considered an easy RB category set, where only one feature is used to indicate category membership, and participants can achieve perfect performance by using a single-dimensional rule. The rule that would result in perfect performance in Figure 1 is "Black shapes belong in Category A, white shapes belong in Category B" because the feature used to indicate category membership is color. Previous research has demonstrated that the Type I category set is the easiest set to learn among younger adults and results in the highest performance (Nosofsky, Gluck, Palmeri, McKinley, \& Glauthier, 1994; Shepard et al., 1961). Type II is considered a hard RB category set, where two features are used to indicate category membership, and participants can achieve perfect performance using a disjunctive rule. The verbal rule that would result in perfect performance in Figure 1 would be "Black triangles
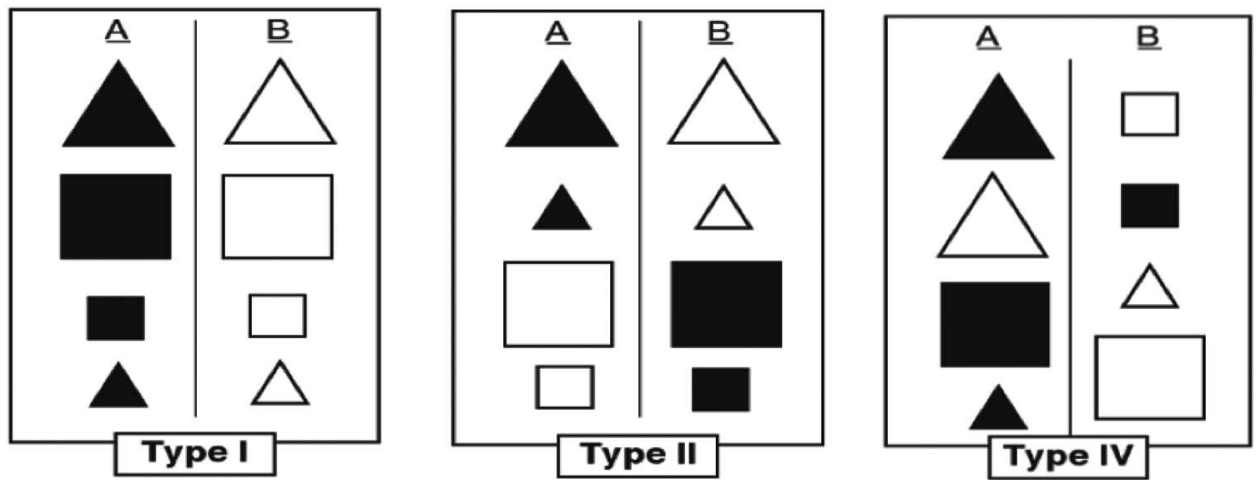

Figure 1. Category learning tasks from Shepard, Hovland, and Jenkins (1961): Type I (easy rule-based), Type II (hard rule-based), and Type IV (nonrule-based). 
and white squares belong in Category $\mathrm{A}$, white triangles and black squares belong in Category B." Therefore, neither color nor shape is individually useful in assigning category membership, but the combination of color and shape is. Type II is considered the second easiest set to learn out of the six category sets, despite the increased logical complexity of the rule. Type IV is considered a family resemblance category set, where all three features are used to indicate category membership. This means that the members of Category A have features in common with one another, for example in Figure 1, they are mostly large, mostly black, and mostly triangles, whereas Category B members are mostly small, mostly white, and mostly squares. This task can be learned by looking at the overall similarity of stimuli, and thus does not require the abstraction and use of a rule. However, the Type IV category set can also be construed as a rule-plus-exception category learning task because another possible method of achieving perfect performance is to memorize the exceptional outlying stimuli (in Figure 1 , this is the big white triangle and the small black square). The verbal rule would be "big shapes (except the white square), plus the small black triangle belong in Category A, and small shapes (except the black triangle), plus the large white square belong in Category B." The Type IV category set is considered the third hardest to learn out of the six category sets.

As mentioned earlier, performance on the SHJ tasks has been examined in many different populations, including children (Minda et al., 2008), monkeys (Smith, Minda, \& Washburn, 2004), and in individuals with depression (Nadler, 2013). Additionally, SHJ tasks have been studied in relation to unsupervised category learning (Love, 2002), working memory capacity (Lewandowsky, 2011), and stimulus composition (Love \& Markman, 2003; Mathy \& Bradmetz, 2011). An important next step is to examine SHJ performance in older adults. Interestingly, in contrast to the Type II advantage typically found in SHJ studies, prior research has shown that children and monkeys actually find Type II harder to learn than Type IV (Minda et al., 2008; Smith et al., 2004). Minda and colleagues (2008) speculated that children struggled with Type II learning because the brain areas thought to mediate the explicit rule-based system and working memory are not fully developed in children. Similarly, Smith et al. (2004) suggested that monkeys struggled with Type II learning because of their smaller prefrontal cortex and lack of verbal abilities.

Based on the research reviewed above, we predict that older adults will perform similarly to younger adults on Type I categories. However, we predict that older adults will struggle to learn Type II categories compared to younger adults, because executive function declines with age. Furthermore, because the disjunctive RB Type II category set heavily relies on executive functioning abilities to test rules, inhibit incorrect rules, and maintain rules in working memory, older adults are predicted to be at a disadvantage at learning this category set, relative to younger adults. Lastly, the Type IV category set can be learned via family resemblance or a complicated rule (i.e., rule-plus-exception). Given past research demonstrating that older adults struggle with learning rule-plusexception categories (Davis et al., 2012) and the fact that stimulus dimensions in the Type IV category set are intercorrelated encouraging family resemblance-based learning, it is predicted that older adults will perform better on the Type IV category set relative to Type II because older adults are expected to have access to the cognitive processes that allow for family resemblance leaning strategies.

In terms of the relationship between category learning and executive functioning abilities, it is predicted that better executive function (i.e., a larger working memory capacity and stronger inhibitory control abilities) will be associated with higher performance on the two rule-based category sets, Types I and II. Because the disjunctive RB category set is thought to rely most heavily on executive functioning, the strongest relationship with executive functioning abilities should occur for this category set.

\section{Method}

\section{Participants}

Participants included 35 younger adults ( $M=18.34$ years; eight males and 27 females) from the University of Western Ontario who participated for course credit and 34 older adults between the ages of 65 and 85 ( $M=71.44$ years; six males and 28 females) recruited from the Kiwanis Seniors' Community Centre and the Boys and Girls Club recreational facility. Older adults received \$20 for participating in the study. Participants were fluent in English and had normal or corrected to normal vision. Information about age, health, and vision was collected via demographic questionnaire that appears as an Appendix. Participants were excluded from the study if they indicated that they had a history of neurological disorders, psychiatric illness, substance abuse, a cerebral vascular event, head trauma, and/or any other neurological conditions.

\section{Materials}

Category learning task. Three category learning tasks were chosen from the original set of six created by Shepard, Hovland, and Jenkins (1961). In each category set there are three features (shape, size, and color) that can have one of two dimensions (square or triangle, large or small, black or white), as shown in Figure 1. In each category set there are eight stimuli, and four belong in each of two separate categories. There were 80 trials $(10$ blocks) total per category set. The Type I set was a singledimensional category with one of the three features acting as the single-dimensional rule. The Type II set was a disjunctive rule category set with two of the three features relevant for the disjunctive rule. The Type IV set was a family resemblance category set in which each category member shared the majority of its features with the other category members and all the features were relevant. All category sets were counterbalanced across participants such that some participants were presented with a Type I set for which color was the relevant dimensions, others were presented with a set for which size was the relevant dimension, and so on.

Memory tasks.

Digit span. Participants heard a recording of a two-digit number sequence at a rate of approximately one digit per second, and the participants were asked to repeat the sequence back to the experimenter in the same order. Participants heard three sequences at each sequence length and as long as they repeated at least one of them correctly they continued on to the next sequence length, for a maximum length of 10 digits. The task was over once the participant was unable to repeat any of the sequences at a given 
length. The procedure for the backward digit span was the same as that for the forward digit span except that the participant was required to recall the digits in reverse order so that the last number was said first and the first number was said last, for a maximum of eight digits. The task was scored as the total number of correct responses.

Inhibitory control tasks.

Flanker task. A version of the Flanker task adapted from Botvinick, Nystrom, Fissell, Carter, and Cohen (1999) was used. A set of five arrows was presented in a row on the computer screen and participants were asked to indicate the direction of the central arrow (target). The target was flanked by two identical arrows on either side (distractors) that were either pointing in the same direction (congruent trial) or the opposite direction (incongruent trial) of the target arrow. The task consisted of 60 trials (30 congruent and 30 incongruent) presented in randomized order. Prior to the experiment participants received five practice trials that were not analyzed. The difference in mean reaction time (RT) between correct responses on congruent and incongruent trials (i.e., a difference score) was used as a measure of inhibitory control. Larger difference scores were indicative of less efficient interference control.

Simon task. In the Simon task, participants were first presented with a fixation cross in the center of the screen (Simon \& Rudell, 1967). Immediately after the cross had disappeared, participants were instructed to press the left key in response to the red circle or the right key in response to a blue circle as fast as possible, regardless of stimulus location. The timing began with the onset of the stimulus, and the response terminated the stimulus. On congruent trials, the stimulus location was on the same side as the required response and on incongruent trials the stimulus location was on the opposite side of the required response. The whole task consisted of 64 trials ( 32 congruent trials and 32 incongruent trials) presented in randomized order to each participant. Prior to the experiment, participants received five practice trials that were not analyzed. Difference scores were calculated by computing the difference in mean RT between correct responses on congruent and incongruent trials.

Stroop task. In the Stroop task (Stroop, 1935), participants were instructed to indicate, as quickly and accurately as possible, whether each word presented on the computer screen was written in red, blue, green, or yellow ink using the properly labeled response buttons. Participants were instructed to ignore the meaning of the words and to focus on the ink color only. The timing began with the onset of the word, and the response terminated the stimulus. Participants first completed 12 practice trials, with accuracy feedback after each trial. The actual task consisted of 72 trials without feedback: 24 congruent trials (i.e., "RED" in red ink), 24 incongruent trials (i.e., "RED" in blue ink) and 24 neutral trials (i.e., noncolor word names like "TREE"). Difference scores were calculated by computing the difference in mean RT between correct responses on congruent and incongruent trials.

Wechsler Abbreviated Scale of Intelligence (WASI) Test. Standardized scores on the WASI vocabulary and matrix reasoning subtests (Wechsler, 1999) were used to calculate the full-scale IQ. WASI subtests were used to provide estimates of verbal and nonverbal intelligence.

\section{Procedure}

Participants were tested individually across two testing sessions, approximately 1 week apart. Younger adults were tested in the Categorization Lab at the University of Western Ontario. Older adults were tested in a quiet room in the senior center. Participants completed all three (Types I, II, and IV) SHJ category sets in one of three orders: I/II/IV, II/IV/I or IV/I/II. A pilot study with 52 university students confirmed that the order of the SHJ category sets did not have an effect on the categorization performance. Participants were told that they would be presented with abstract shapes and asked to classify them as belonging to category A or category B. Participants saw each stimulus one-at-a-time on the computer screen and were instructed to press the button labeled "A" or "B" to indicate whether each shape belonged in category A or B respectively. After responding, participants were given corrective feedback (the words "correct" or "incorrect" appeared above the stimulus object). Another trial began following this feedback. Stimuli were presented in random order within each block of eight and blocks were presented in an unbroken fashion. Following completion of the first category set, participants completed the second and third category set. Before completing the next two category sets, participants were told that even though the objects would look the same as before, the category set is different and they should adopt a new strategy. Participants were told that they could take a break between category sets if they wished.

During the second testing session, participants first completed three inhibitory control tasks: the Flanker task, Simon task, and Stroop task. Following the Stroop task, participants received a short break, after which they were administered the forward and backward digit span. Lastly, participants completed the WASI. Each testing session lasted approximately one hour.

\section{Results}

\section{Category Learning}

The average categorization performance of younger and older adults across the three SHJ category sets is displayed in Figure 2.

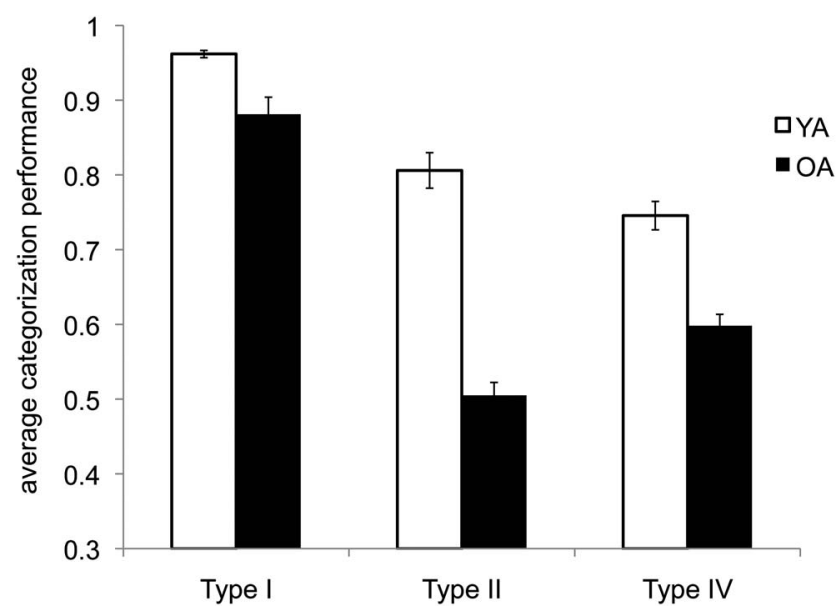

Figure 2. Average categorization performance of younger adults (YA) and older adults (OA) across the 10 learning blocks. 
The learning curve of the younger adults is similar to one originally reported by Shepard et al. (1961), with the Type I category set having the highest performance, followed by Type II, and the Type IV category set. Similar to younger adults, older adults found Type I the easiest. However, unlike younger adults, older adults performed worse on Type II relative to Type IV. Learning curves for each age group and category set across learning blocks is displayed in Figure 3. A 3 (Category Type: Type I, II, IV) $\times 2$ (Age: younger, older) $\times 10$ (Blocks) mixed analysis of variance (ANOVA) was conducted to further examine how younger and older adults learned the three category sets. If the sphericity assumption was violated, ( $p<.05$, Mauchly's test of sphericity), a Greenhouse-Geisser correction was performed.

Results revealed a significant main effect of category type, $F(2$, $134)=151.42, p<.001, \eta^{2}=.69$, power $=1.00$, as well as a main effect of age, $F(1,67)=111.45, p<.001, \eta^{2}=.63$, power $=1.00$. There was also a main effect of block, $F(7,439)=$ $72.15, p<.001, \eta^{2}=.52$, power $=1.00$. Additionally, there was a signification interaction between category type and age group, $F(2,130)=21.88, p=<.001, \eta^{2}=.25$, power $=1.00$, The Category Type $\times$ Age Group interaction is of particular interest, because it demonstrates a crossover effect, where younger adults perform better on Type II compared to Type IV and older adults show the reverse effect, performing better on Type IV compared to Type II (see Figure 2). Lastly, there was a three-way interaction between age, category, and block, $F(12,763)=3.93, p=<.001$, $\eta^{2}=.06$, power $=1.00$. In order to further explore the three-way type interaction, three separate analyses of variance were conducted (one for each of the three category sets).

Type I categorization performance. For the Type I (singledimensional rule) category set, there was a main effect of age. The Type I categorization performance of older adults was significantly lower than younger adults, $F(1,67)=12.20, p=.001$, $\eta^{2}=.99$, power $=.93$. There was a significant main effect of block, $F(4.4,296)=50.14, p<.001, \eta^{2}=.43$, power $=1.00$ [Greenhouse-Geisser corrected], suggesting that learning occurred across the blocks. There was also a significant interaction between age and block, $F(4.4,296)=2.93, p=.02, \eta^{2}=.04$, power $=$ .81 , demonstrating that younger adults learned the Type I category set faster than older adults.

Type II categorization performance. For the Type II (disjunctive rule) category set, there was a main effect of age, $F(1$, $67)=103.34, p<.001, \eta^{2}=.61$, power $=1.00$, suggesting that younger adults outperformed older adults. There was also a main effect of block, $F(7.3,494)=19.64, p<.001, \eta^{2}=.23$, power $=$ 1.00 [Greenhouse-Geisser corrected]. A significant age $\mathrm{x}$ block interaction was also found, $F(7.3,494)=5.61, p<.001, \eta^{2}=.08$, power $=1.00$ [Greenhouse-Geisser corrected], showing that younger adults learned the Type II category set faster than older adults.

Type IV categorization performance. For the Type IV (family resemblance) category set, there was a main effect of age, $F(1$, $67)=36.19, p<.001, \eta^{2}=.35$, power $=1.00$, with younger adults outperforming older adults. There was a main effect of block $F(6.8,454)=17.73, p<.001, \eta^{2}=.21$, power $=1.00$ [Greenhouse-Geisser corrected]. Lastly, there was a significant Age $\times$ Block interaction, $F(6.8,454)=3.33, p=.002, \eta^{2}=.05$, power $=.96$ [Greenhouse-Geisser corrected], suggesting that younger adults learned the Type IV category set faster than older adults.

Order effects. To ensure that order effects were not present, three separate ANOVAs were conducted for each of the category sets, examining each of the three randomized orders (i.e., I/II/IV, II/IV/I or IV/I/II). This analysis was done to eliminate the possibility that some participants performed better on certain category sets than other participants, because they completed certain category sets first. There were no order effects for the Type I category set, $F(2,63)=.63, p=.54$, the Type II category set, $F(2,63)=$ $.36, p=.70$, or the Type IV category set, $F(2,63)=.06, p=.94$. This means that performance on the three category sets were not impacted by whether participants received Type I, Type II or Type $\mathrm{IV}$, first, in the middle, or last.

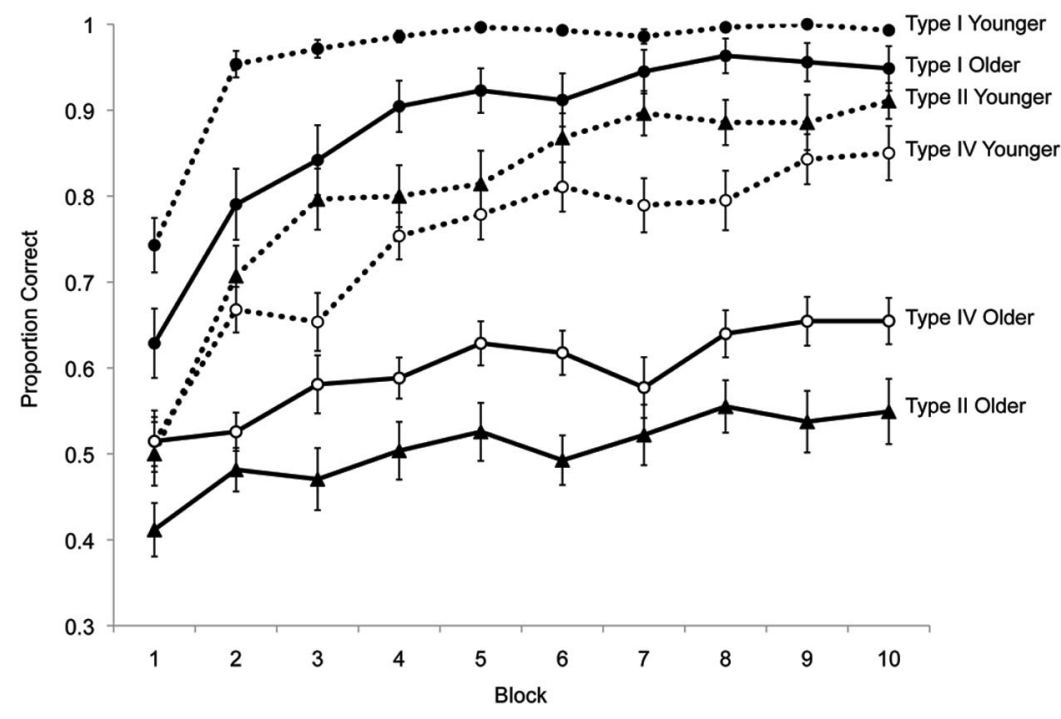

Figure 3. Categorization performance of younger and older adults across learning blocks in each of the three category sets. Error bars denote the standard error of the mean. 


\section{Strategy Analysis}

In addition to category learning performance, we were also interested in whether participants used a single-dimensional rule strategy in learning any of the three category sets. This is important to know because in many cases, what might appear to be moderate performance on the Type IV family resemblance category set might actually be a result of participants learning a suboptimal single-dimensional rule (e.g., attention to a single dimension in the Type IV category set would result in $75 \%$ correct). The same type of strategy analysis was performed by Minda et al. (2008) when examining SHJ learning in children.

For each participant, we identified the response made (either Category A or B) for each stimulus. Next, we calculated for each block the correlation between the value of each dimension (e.g., square or triangle) and the response. If a participant responded to a single dimension, then the correlation between stimulus and response would be 1.0 regardless of which category that participant was learning. This analysis would indicate if a participant had adopted a single-dimensional rule, even if the rule was suboptimal. Following the correlational analysis, we counted how many participants displayed at least two blocks (including nonconsecutive blocks) of perfect rule-response correlations. As Table 1 shows, we typically observed single-dimensional responding only in Type I categories, with the exception of four older adults who never consistently applied a single-dimensional rule-based strategy when completing the Type I category set. Zero younger adults and only two of the 34 older adults showed a single-dimensional performancedimension correlation for the Type II categories. The fact that older adults were performing at chance on Type II category set, yet the majority was not fit by a single-dimensional rule, suggests that older adults frequently switched their strategies throughout the task. Given the fact that one could only achieve $50 \%$ by applying a single-dimensional rule in the Type II category set, it makes sense that older adults did not consistently apply a single-dimensional rule, but rather switched rules to avoid negative feedback. Lastly, eight of the 35 younger adults and nine of the 34 older adults showed a single-dimensional performance-dimension correlation for the Type IV category set, suggesting that roughly a quarter of participants (both younger and older adults) relied on singledimensional rules to learn the Type IV category set. ${ }^{1}$ It should also be noted, that these participants were fit by a single-dimensional rule across at least two learning blocks, in the Type IV condition. The majority of these participants were fit by a single-dimensional rule quite early in the task, and did not persist in using a singledimensional strategy for more then two learning blocks. Furthermore, implying that they most likely used a family resemblance based strategy for the remainder of the task. ${ }^{2}$

Table 1

Percentage of Participants Using Single-Dimensional Rules

\begin{tabular}{lccc}
\hline \multicolumn{1}{c}{ Age group } & Type I & Type II & Type IV \\
\hline Younger adults & $100 \%$ & $0 \%$ & $23 \%$ \\
Older adults & $88 \%$ & $6 \%$ & $26 \%$ \\
\hline
\end{tabular}

Note. A total of 35 younger adults and 34 older adults completed the study.

\section{Executive Functioning and IQ}

Mean scores of younger and older adults on the inhibitory control and working memory measures were compared. There was a significant difference between younger $(M=57.41, S D=55.26)$ and older adults $(M=1,183.36, S D=196.50)$ on the Stroop task, $t(38)=-3.6, p=.001$. Younger adults $(M=19.45, S D=2.90)$ outperformed older adults $(M=17.73, S D=2.77)$ on the forward digit span task, $t(67)=2.52, p=.014$. Younger adults $(M=12.2$, $S D=3.88)$ also outperformed older adults $(M=9.71, S D=3.28)$ on the backward digit span task, $t(66)=2.88, p=.005$. There was no significant difference between younger $(M=45.94, S D=$ 22.04) and older adults $(M=46.42, S D=31.13)$ on the Flanker task, $t(57)=-.07, p=.94$. There was also no significant difference between younger $(M=38.72, S D=30.24)$ and older adults $(M=56.80, S D=48.17)$ on the Simon task, $t(55)=-1.85$, $p=.07$.

To examine the relationship between category learning performance and executive functioning abilities in younger and older adults, correlational analyses were conducted. Average categorization performance across the last five learning blocks was correlated with the different measures of inhibitory control and working memory. For both younger and older adults, the only signification correlation found was between Type II categorization performance and backward digit span (see Tables 2 and 3). This suggests that having a larger working memory capacity is advantageous for learning Type II (complicated) RB category sets. Furthermore, the lack of correlations between executive functioning measures (Stroop, Flanker, and Simon) and Type I and Type II performance is not surprising, given the lack of variability in categorization performance scores. The majority of younger and older adults learned the Type I category set, with the exception of a few older adults. In contrast, most younger adults learned the Type II category set but few older adults did. No correlations were expected between Type IV categorization performance and executive functioning measures, because Type IV category learning is thought to rely less heavily on executive functioning compared to Types I and II. When controlling for age, partial correlations revealed that backward digit span correlated with both Type II, $r=.318, p=$ .009 performance and Type IV performance, $r=.260, p=.035$. No other correlations were significant. The partial correlational analyses revealed that when age is controlled for, participants with greater working memory capacities perform better on the Type II and Type IV category sets.

\footnotetext{
${ }^{1}$ Roughly $75 \%$ of both younger and older adults were not employing a single-dimensional rule in the Type IV category set. This analysis does not exclude the possibility that participants may have learned the Type IV categories via a multidimensional rule. However, given the low dimensionality of the FR categories, a multidimensional rule might be difficult to distinguish from family resemblance responding. We favor the conclusion that most older adults relied on a family resemblance strategy to solve the Type IV category set, because given their difficulty learning the Type II disjunctive rule-based category set, it is unlikely that older adults would successfully be able to apply a complex, multidimensional rule-based strategy when learning the Type IV category set.

${ }^{2}$ When a stricter criterion based on three learning blocks rather than two was used to identify single-dimensional rule users, there was no evidence of single-dimensional rule use, suggesting that if subjects were using a single-dimensional rule in Type IV, they did not appear to use it consistently.
} 
Table 2

Intercorrelations Among the Study Variables for Younger Adults

\begin{tabular}{lccc}
\hline \multicolumn{1}{c}{ Variable } & Type I & Type II & Type IV \\
\hline 1. Age (months) .061 & .165 & -.036 & \\
2. Forward digit span & .203 & -.053 & .253 \\
3. Backward digit span & .132 & $.325 \dagger$ & .303 \\
4. Flanker difference score & .031 & -.138 & -.104 \\
5. Simon difference score & .012 & -.163 & -.176 \\
6. Stroop difference score & -.186 & .024 & .301 \\
\hline
\end{tabular}

Note. Age, inhibitory control and working memory measures were correlated with average Type I, Type II, and Type IV categorization performance over the last five learning blocks.

$\dagger p<.06$.

In order to examine more closely the relationship between category learning and digit span, we conducted a partial correlation to examine the relationship between Type II performance and backward digit span, controlling for forward digit span. For younger adults, the relationship was significant, $r=.44, p=.009$. For older adults, the relationship between Type II performance and backward digit span was no longer significant, $r=.28, p=.10$, two-tailed. This suggests that the lower performance on Type II categories by older adults may not be purely a result of a decline in working memory performance.

A $t$ test was conducted to determine whether younger and older adults differed on IQ scores. Results showed that older adults $(M=117, S D=14.4)$ had a significantly higher IQ score compared to younger adults $(M=109, S D=7.5), t(49)=-2.8$, $p=.007$. However, this effect was driven by the fact that older adults performed much better on the Vocabulary subtest of the WASI compared to younger adults, most likely due to increased life experience. This is not a concern, given that younger adults still outperformed older adults on all three SHJ category sets, ruling out the possibility that the IQ difference influenced category learning performance between the groups.

Among older adults, IQ was not correlated with average categorization performance over the last five blocks on the Type I, $r=-.09, p=.62$, Type II, $r=.17, p=.34$, or Type IV, $r=.33$, $p=.06$ category set. Among younger adults, IQ was not correlated with the average categorization performance over the last five blocks on the Type I, $r=-.21, p=.24$ and Type II, $r=.28, p=$ .10 category set. Type IV did correlate with IQ in younger adults, $r=.51, p=.002$, though we made no specific prediction about this relationship and did not analyze it further.

\section{Discussion}

The current study examined the relationship between executive functioning and performance on three different types of category learning tasks: an easy RB task (Type I), a complex disjunctive RB task (Type II), and a FR task (Type IV). Category learning differences between younger and older adults revealed that while both age groups performed similarly when learning Type I, older adults struggled significantly more than younger adults when learning Type II. With the majority of younger adults learning the Type II category set, and almost all older adults performing at chance, it was clear that older adults had difficulty discovering the more complex rule. Younger adults also outperformed older adults on the Type IV category set, which required adopting an implicit, overall similarity type strategy.

Findings from the current study share many similarities with findings from Minda et al.'s (2008) study examining SHJ learning in children. Contrary to prior research with younger adults usually showing a Type II advantage over Type IV (Shepard et al., 1961; Nosofsky et al., 1994; Smith et al., 2004), present findings along with those from Minda and colleagues (2008) have demonstrated a reversal in learning, with Type IV being learned significantly better than Type II by older adults and children. This reversal of the traditional SHJ ordering is quite interesting, and may shed some light on the role of the prefrontal cortex and working memory on different types of category learning. Similar to our older adults, children in the Minda et al. (2008) study performed comparably well when learning the Type I category set because the rule was based on a simple, single-dimensional rule. Even though the areas that mediate the explicit rule-based system are not fully developed in children (Bunge \& Zelazo, 2006), the singledimensional rule is easy to find and verbalize with a single proposition and places minimal demands on hypothesis testing and working memory abilities. We draw similar conclusions in regards to our findings with older adults. Functioning of the prefrontal cortex is known to decline with age, however, given the relative simplicity of the Type I rule, older adults are still able to learn this category set quite well. Even though younger adults ( $96 \%$ correct) performed significantly better than older adults ( $88 \%$ correct) on the Type I category set, older adults still demonstrated high performance on this category set. Older adults' lower Type I performance relative to younger adults is most likely the result of a lapse in memory. Prior literature suggests that it is common for older adults to learn a task/rule fairly well, but at times experience dips in performance due to memory lapses (West, 2001; West, Murphy, Armilio, Craik, \& Stuss, 2002). Often referred to as transient goal neglect, older adults experience periods of active rule maintenance failure, rather than a difficulty actively maintaining the appropriate rule. Because there were eight trials per learning block, older adults periodically made one error during the learning block (i.e., which equates to $88 \%$ correct).

Minda et al.'s (2008) findings offer an intriguing parallel to the present research. Similar to children in the Minda et al. study, older adults in our study demonstrated difficulty learning the Type II disjunctive rule-based category set. Minda et al. attributed children's difficulty in learning the Type II category set to an underdeveloped explicit rule system. That is, Type II category learning

Table 3

Intercorrelations Among the Study Variables for Older Adults

\begin{tabular}{lrrr}
\hline \multicolumn{1}{c}{ Variable } & Type I & Type II & Type IV \\
\hline 1. Age (months) & -.142 & -.164 & .105 \\
2. Forward digit span & .014 & -.227 & .163 \\
3. Backward digit span & .040 & $.353^{*}$ & .252 \\
4. Flanker difference score & .187 & .140 & -.281 \\
5. Simon difference score & .289 & -.071 & -.236 \\
6. Stroop difference score & .076 & -.171 & -.042 \\
\hline
\end{tabular}

Note. Age, inhibitory control and working memory measures were correlated with average Type I, Type II, and Type IV categorization performance over the last five learning blocks.

$* p<.05$. 
requires more complex verbal rules, relative to the single propositional rule required in the Type I category set. As a result, children do not fully possess the executive functioning abilities required for learning this more complex category set. Working memory and executive functioning abilities are known to decline with age (Peters, 2006; West, 1996), which may have led to older adult's difficulty learning the Type II category set in the current study. Along the same lines, Smith et al. (2004) found that Type II was the second easiest for young adults but was the second most difficult category set for monkeys. They attributed this difficulty to the fact that monkeys have a much smaller prefrontal cortex and no verbal abilities, which are key for Type II learning.

Lastly, the present results also differed from the findings of Minda and colleagues (2008) showing that younger adults and children performed similarly on the Type IV category set. The current study demonstrated that older adults significantly underperformed younger adults on Type IV, suggesting that the correspondence between younger children and older adults does not extend to every task. Minda et al. suggest that children and younger adults perform comparably on Type IV because family resemblance learning is mediated by areas that are equally developed in both children and adults. Our findings are in line with research by Filoteo and Maddox (2004) and Maddox et al. (2010) showing that older adults struggled with learning several varieties of nonrule defined category sets. Filoteo and Maddox suggested that older adults were more likely to adopt a rule strategy that was not optimal for learning information-integration categories. Maddox et al. (2010) suggested that this deficit in FR category learning may be because older adults find it more difficult to transition from the default, explicit RB system to the implicit FR system. Furthermore, younger adults may have performed better than older adults on the Type IV category set in the current study because executive functioning abilities are required to inhibit the explicit RB system, and switch over to the implicit overall-similarity based system. However, the Maddox et al. (2010) FR category set (four category sets with lines varying across length and orientation) was quite different from our category set (two category sets with shapes varying along three dimensions), so direct comparisons may not be possible, because it is also possible to learn our Type IV category set with a complicated rule rather than strictly through FR learning. Additionally, given that research has shown that implicit learning takes longer than explicit, RB learning (Ashby, Alfonso-Reese, Turken, \& Waldron, 1998), it may be the case that older adults needed more time to discover the correct implicit-based strategy. If given more trials to complete, older adults may have begun to perform more similarly to younger adults.

Our key finding is that older adults found the Type II categories (a complex, disjunctive rule) more difficult to learn than the Type IV categories (a family resemblance set). This is contrary to findings examining category learning in younger adults, which has shown that Type IV is more difficult than Type II. The finding that older adults struggled more with the Type II category set highlights the fact that executive functioning may be responsible for the performance differences in category learning between younger and older adults. Results showed that among both younger and older adults, a larger working memory capacity (as measured by the backward digit span) was associated with better Type II category learning performance. Additionally, when controlling for age, we found that that working memory capacity was associated with
Type II and Type IV categorization performance. This suggests that working memory may be important for learning disjunctive rules, and possibly also for speeding up hypothesis testing so individuals can switch between systems. The fact that a relationship was not found between inhibitory control abilities and category learning can mean either two things: inhibitory control is not necessary for learning certain types of categories or alternatively, that the lack of variability in categorization performance scores between each age group, masked important effects. Given a different category set with more variability in performance, where a subset of older adults were performing well and others were not, we might see a relationship between inhibitory control and category learning emerge. The current findings do suggest that Type II category learning may place a higher demand on working memory and not so much executive functioning abilities like inhibitory control. Overall, these results support previous research showing that older adults struggle with both RB and nonrule-based FR category learning (Filoteo \& Maddox, 2004; Racine et al., 2006; Davis et al., 2012; Maddox et al., 2010).

It is possible that if additional learning trials were added to the Type II category set, older adults might have improved to a level that is comparable to younger adults. This would not have changed our interpretation of our results for two reasons. First, even with extended training, we would have still observed the reversal in rank order difficulty between Type II and Type IV. Second, our primary conclusion was that it is the reduced working memory capacity associated with cognitive aging that brings about the learning differences. This would still hold even if the additional trials allowed for eventual mastery of the category set.

In addition to the explicit (Types I and II)/implicit (Type IV) distinction often used to describe the SHJ category sets, Boolean complexity is another way of conceptualizing the different types of categories. That is, SHJ types can be considered from the perspective of mathematical logic, where Boolean complexity refers to the length of the shortest logically equivalent propositional formula. Furthermore, Feldman (2000) demonstrated that the subjective difficulty of the category set is directly proportional to its Boolean complexity, with Type I being the easiest, followed by Type II, and Type IV being the hardest. That is, the Type I structure requires attention to only one dimension and is easiest to learn. Type II requires attention to two dimensions and is the next easiest to learn. Lastly, Type IV requires attention to all three dimensions and is considered by many to be the hardest to learn. Using this logic, Goodman, Tenenbaum, Feldman, and Griffiths (2008) proposed a rational rules model that combines logical rule induction with Boolean complexity. This model predicts that Type II could be more difficult to acquire under certain conditions-when the participant or experimental setting favors unidimensional rules. This makes sense, as applying a unidimensional rule when learning the Type II category set would result in chance performance. That being said, with respect to Boolean complexity, it is quite impressive that older adults learned the harder Type IV category set better than the easier Type II category set. Even though our strategy analysis findings did not indicate that older adults were heavily relying on single-dimensional rules to learn the Type II category set, we suspect that older adults relied on single-dimensional rules more so than younger adults. The reason being that applying a single-dimensional rule during Type II learning would result in a large number of errors. It is unlikely that older adults would 
internalize this negative feedback and continue to apply a singledimensional rule that resulted in numerous errors. The most logical alternative is that older adults applied single-dimensional rules during Type II learning, but frequently switched rules during the course of the task to avoid negative feedback.

Weighing all possible conclusions, it seems more likely that the reason why older adults did not demonstrate a Type II advantage is because Type II learning places the heaviest demands on cognitive resources (i.e., working memory load) and it is not as intuitive as the other category sets. That is, Type I is relatively simple to learn because it involves identification of a straightforward single dimensional rule, placing minimal demands on hypothesis testing and working memory abilities, and is encountered quite frequently in everyday life. Second, Type IV is considered the next easiest category set for older adults to learn because of its family resemblance structure, which is reminiscent of natural categories (Rosch \& Mervis, 1975). Again, minimal working memory abilities are required to identify the overall similarity structure of the Type IV category set, realizing which features most of the category members have in common with each other. In contrast, Type II learning requires a high degree of verbal working memory to acquire and combine rules together to arrive at the correct rule. Due to declines in verbal working memory with normal aging, it is possible that the Type II category representation was not actively acquired and maintained in working memory in a manner which would allow older adults to apply the disjunctive rule accurately. This conclusion is consistent with theories of age-related impairment in working memory (Craik, Morris, \& Glick, 1990), stating that older adults struggle to test various rules and maintain this information in working memory. These findings suggest that small declines in working memory capacity relative to younger adults may have a big impact on the complex rule-based category learning abilities of older adults. It is clear that future research is required to identify the importance of working memory in older adults' ability to learn disjunctive rule-based category sets. Given the fact that individuals of all ages rely on Type II, disjunctive rule-based learning in day-to-day life, it is important to understand the cognitive mechanisms involved.

\section{References}

Ashby, F. G., Alfonso-Reese, L. A., Turken, A. U., \& Waldron, E. M. (1998). A neuropsychological theory of multiple systems in category learning. Psychological Review, 105, 442-481. http://dx.doi.org/10 .1037/0033-295X.105.3.442

Axelrod, B. N., \& Henry, R. R. (1992). Age-related performance on the Wisconsin card sorting, similarities, and controlled oral word association tests. Clinical Neuropsychologist, 6, 16-26. http://dx.doi.org/10.1080/ 13854049208404113

Bopp, K. L., \& Verhaeghen, P. (2005). Aging and verbal memory span: A meta-analysis. The Journals of Gerontology Series B, Psychological Sciences and Social Sciences, 60, 223-233. http://dx.doi.org/10.1093/ geronb/60.5.P223

Botvinick, M., Nystrom, L. E., Fissell, K., Carter, C. S., \& Cohen, J. D. (1999). Conflict monitoring versus selection-for-action in anterior cingulate cortex. Nature, 402, 179-181. http://dx.doi.org/10.1038/46035

Braver, T. S., Barch, D. M., Keys, B. A., Carter, C. S., Cohen, J. D., Kaye, J. A., . . . Reed, B. R. (2001). Context processing in older adults: Evidence for a theory relating cognitive control to neurobiology in healthy aging. Journal of Experimental Psychology: General, 130, 746763. http://dx.doi.org/10.1037/0096-3445.130.4.746
Bunge, S. A., \& Zelazo, P. D. (2006). A brain-based account of the development of rule use in childhood. Current Directions in Psychological Science, 15, 118-121. http://dx.doi.org/10.1111/j.0963-7214.2006 .00419.x

Butler, K. M., Zacks, R. T., \& Henderson, J. M. (1999). Suppression of reflexive saccades in younger and older adults: Age comparisons on an antisaccade task. Memory \& Cognition, 27, 584-591. http://dx.doi.org/ 10.3758/BF03211552

Chasseigne, G., Mullet, E., \& Stewart, T. R. (1997). Aging and multiple cue probability learning: The case of inverse relationships. Acta Psychologica, 97, 235-252. http://dx.doi.org/10.1016/S00016918(97)00034-6

Craik, F. I. M., Morris, R. G., \& Glick, M. L. (1990). Adult age difference in working memory. In G. Vallar \& T. Shallice (Eds.), Neuropsychological impairments of short-term memory (pp. 247-267). Cambridge, United Kingdom: Cambridge University Press. http://dx.doi.org/10 .1017/CBO9780511665547.014

Davidson, D. J., Zacks, R. T., \& Williams, C. C. (2003). Stroop interference, practice, and aging. Neuropsychology, Development, and Cognition Section B, Aging, Neuropsychology and Cognition, 10, 85-98. http://dx.doi.org/10.1076/anec.10.2.85.14463

Davis, T., Love, B. C., \& Maddox, W. T. (2012). Age-related declines in the fidelity of newly acquired category representations. Learning \& Memory, 19, 325-329. http://dx.doi.org/10.1101/lm.024919.111

Feldman, J. (2000). Minimization of Boolean complexity in human concept learning. Nature, 407, 630-633. http://dx.doi.org/10.1038/35036586

Filoteo, J. V., \& Maddox, W. T. (2004). A quantitative model-based approach to examining aging effects on information-integration category learning. Psychology and Aging, 19, 171-182.

Gathercole, S. E. (1999). Cognitive approaches to the development of short-term memory. Trends in Cognitive Sciences, 3, 410-419. http:// dx.doi.org/10.1016/S1364-6613(99)01388-1

Goodman, N. D., Tenenbaum, J. B., Feldman, J., \& Griffiths, T. L. (2008). A rational analysis of rule-based concept learning. Cognitive Science, 32, 108-154.

Gorlick, M. A., Giguère, G., Glass, B. D., Nix, B. N., Mather, M., \& Maddox, W. T. (2013). Attenuating age-related learning deficits: Emotional valenced feedback interacts with task complexity. Emotion, 13, 250-261. http://dx.doi.org/10.1037/a0030071

Greenwood, P. M. (2000). The frontal aging hypothesis evaluated. Journal of the International Neuropsychological Society, 6, 705-726. http://dx .doi.org/10.1017/S1355617700666092

Grieve, S. M., Williams, L. M., Paul, R. H., Clark, C. R., \& Gordon, E. (2007). Cognitive aging, executive function, and fractional anisotropy: A diffusion tensor MR imaging study. American Journal of Neuroradiology, 28, 226-235.

Hayslip, B., Jr., \& Sterns, H. L. (1979). Age differences in relationships between crystallized and fluid intelligences and problem solving. Journal of Gerontology, 34, 404-414. http://dx.doi.org/10.1093/geronj/34.3 .404

Huang-Pollock, C. L., Maddox, W. T., \& Karalunas, S. L. (2011). Development of implicit and explicit category learning. Journal of Experimental Child Psychology, 109, 321-335. http://dx.doi.org/10.1016/j.jecp 2011.02.002

Kolb, B., Mychasiuk, R., Muhammad, A., Li, Y., Frost, D. O., \& Gibb, R. (2012). Experience and the developing prefrontal cortex. Proceedings of the National Academy of Sciences of the United States of America, 109, 17186-17193. http://dx.doi.org/10.1073/pnas.1121251109

Lewandowsky, S. (2011). Working memory capacity and categorization: Individual differences and modeling. Journal of Experimental Psychology: Learning, Memory, and Cognition, 37, 720-738. http://dx.doi.org/ $10.1037 / \mathrm{a} 0022639$ 
Love, B. C. (2002). Comparing supervised and unsupervised category learning. Psychonomic Bulletin \& Review, 9, 829-835. http://dx.doi.org/ 10.3758/BF03196342

Love, B. C., \& Markman, A. B. (2003). The nonindependence of stimulus properties in human category learning. Memory \& Cognition, 31, 790799. http://dx.doi.org/10.3758/BF03196117

Maddox, W. T., Pacheco, J., Reeves, M., Zhu, B., \& Schnyer, D. M. (2010). Rule-based and information-integration category learning in normal aging. Neuropsychologia, 48, 2998-3008. http://dx.doi.org/10 .1016/j.neuropsychologia.2010.06.008

Mathy, F., \& Bradmetz, J. (2011). An extended study of the nonindependence of stimulus properties in human classification learning. Quarterly Journal of Experimental Psychology: Human Experimental Psychology, 64, 41-64. http://dx.doi.org/10.1080/17470218.2010.493972

May, C. P., \& Hasher, L. (1998). Synchrony effect in inhibitory control over thought and action. Journal of Experimental Psychology: Human Perception and Performance, 24, 363-379.

Miles, S. J., \& Minda, J. P. (2011). The effects of concurrent verbal and visual tasks on category learning. Journal of Experimental Psychology: Learning, Memory, and Cognition, 37, 588-607. http://dx.doi.org/10 $.1037 / \mathrm{a} 0022309$

Minda, J. P., Desroches, A. S., \& Church, B. A. (2008). Learning ruledescribed and nonrule-described categories: A comparison of children and adults. Journal of Experimental Psychology: Learning, Memory, and Cognition, 34, 1518-1533. http://dx.doi.org/10.1037/a0013355 category learning. Psychology of Learning and Motivation, 52, 117-162.

Miyake, A., Friedman, N. P., Emerson, M. J., Witzki, A. H., Howerter, A., \& Wager, T. D. (2000). The unity and diversity of executive functions and their contributions to complex "frontal lobe" tasks: A latent variable analysis. Cognitive Psychology, 41, 49-100. http://dx.doi.org/10.1006/ cogp.1999.0734

Nadler, R. (2013). The influence of mood and motivation on cognitive flexibility. Electronic Thesis and Dissertation Repository. Paper 1528.

Nosofsky, R. M., Gluck, M. A., Palmeri, T. J., McKinley, S. C., \& Glauthier, P. (1994). Comparing models of rule-based classification learning: A replication and extension of Shepard, Hovland, and Jenkins (1961). Memory \& Cognition, 22, 352-369. http://dx.doi.org/10.3758/ BF03200862

Olincy, A., Ross, R. G., Youngd, D. A., \& Freedman, R. (1997). Age diminishes performance on an antisaccade eye movement task. Neurobiology of Aging, 18, 483-489. http://dx.doi.org/10.1016/S01974580(97)00109-7

Park, D. C., Lautenschlager, G., Hedden, T., Davidson, N. S., Smith, A. D., \& Smith, P. K. (2002). Models of visuospatial and verbal memory across the adult life span. Psychology and Aging, 17, 299-320. http://dx.doi .org/10.1037/0882-7974.17.2.299

Peters, R. (2006). Ageing and the brain. Postgraduate Medical Journal, 82, 84-88. http://dx.doi.org/10.1136/pgmj.2005.036665

Rabi, R., \& Minda, J. P. (2014). Rule-based category learning in children: The role of age and executive functioning. PLOS ONE, 9, e85316. http://dx.doi.org/10.1371/journal.pone.0085316

Racine, C. A., Barch, D. M., Braver, T. S., \& Noelle, D. C. (2006). The effect of age on rule-based category learning. Neuropsychology, Devel- opment, and Cognition Section B, Aging, Neuropsychology and Cognition, 13, 411-434. http://dx.doi.org/10.1080/13825580600574377

Raz, N. (2000). Aging of the brain and its impact on cognitive performance: Integration of structural and functional findings. In F. I. M. Craik \& T. A. Salthouse (Eds.), Handbook of aging and cognition II (pp. 1-90). Mahwah, NJ: Erlbaum.

Ridderinkhof, K. R., Span, M. M., \& van der Molen, M. W. (2002). Perseverative behavior and adaptive control in older adults: Performance monitoring, rule induction, and set shifting. Brain and Cognition, 49, 382-401. http://dx.doi.org/10.1006/brcg.2001.1506

Rosch, E., \& Mervis, C. B. (1975). Family resemblances: Studies in the internal structure of categories. Cognitive Psychology, 7, 573-605. http://dx.doi.org/10.1016/0010-0285(75)90024-9

Shepard, R. N., Hovland, C. I., \& Jenkins, H. M. (1961). Learning and memorization of classifications. Psychological Monographs: General and Applied, 75, 1-42.

Simon, J. R., \& Rudell, A. P. (1967). Auditory S-R compatibility: Reaction time as a function of ear-hand correspondence and ear-response-location correspondence. Journal of Applied Psychology, 51, 300-304. http://dx doi.org/10.1037/h0020586

Smith, J. D., Minda, J. P., \& Washburn, D. A. (2004). Category learning in rhesus monkeys: A study of the Shepard, Hovland, and Jenkins (1961) tasks. Journal of Experimental Psychology: General, 133, 398-414. http://dx.doi.org/10.1037/0096-3445.133.3.398

Stroop, J. R. (1935). Studies of interference in serial verbal reactions. Journal of Experimental Psychology, 18, 643-662. http://dx.doi.org/10 $1037 / \mathrm{h} 0054651$

Uylings, H. B., \& de Brabander, J. M. (2002). Neuronal changes in normal human aging and Alzheimer's disease. Brain and Cognition, 49, 268 276. http://dx.doi.org/10.1006/brcg.2001.1500

van der Mollen, M. W., \& Ridderinkhof, K. R. (1998). The growing and aging brain: Life-span changes in brain and cognitive functioning. In A Demetriou, W. Doise, \& C. F. M. van Lieshout (Eds.), Life-span developmental psychology: A European perspective (pp. 35-99). Chichester, United Kingdom: Wiley.

Wechsler, D. (1999). Wechsler Abbreviated Scale of Intelligence (WASI) San Antonio, TX: Psychological Corporation.

West, R. L. (1996). An application of prefrontal cortex function theory to cognitive aging. Psychological Bulletin, 120, 272-292. http://dx.doi.org/ 10.1037/0033-2909.120.2.272

West, R. (1999). Age differences in lapses of intention in the Stroop task. The Journals of Gerontology Series B, Psychological Sciences and Social Sciences, 54, 34-43. http://dx.doi.org/10.1093/geronb/54B.1.P34

West, R. (2001). The transient nature of executive control processes in younger and older adults. The European Journal of Cognitive Psychology, 13, 91-105. http://dx.doi.org/10.1080/09541440042000232

West, R., Murphy, K. J., Armilio, M. L., Craik, F. I., \& Stuss, D. T. (2002) Lapses of intention and performance variability reveal age-related increases in fluctuations of executive control. Brain and Cognition, 49, 402-419. http://dx.doi.org/10.1006/brcg.2001.1507

Williams, B. R., Ponesse, J. S., Schachar, R. J., Logan, G. D., \& Tannock R. (1999). Development of inhibitory control across the life span. Developmental Psychology, 35, 205-213. 


\section{Appendix}

\section{Demographics Questionnaire}

1. Age (years):

2. Sex:

$\square 1$ Male

$\square 2$ Female

3. Years of education (report achieved level using the codes below; if an attempted level is not completed, enter the number of years attended). Codes: High school diploma $=12$; Bachelor's degree $=16$; Master's degree $=18$; Doctorate $=20$ years

4. Do you feel that your memory or thinking skills have gotten worse recently?

$\square$ Yes

$\square$ No

5. Compared to yourself 5 years ago, do you think that your memory is:

$\square 1$ Much better

$\square 2$ Better

$\square 3$ About the same

$\square 4$ Worse

$\square 5$ Much worse

6. In general, how would you rate your health? Would you say it is:

$\square 1$ Excellent

$\square 2$ Very good

$\square 3 \mathrm{Good}$

$\square 4$ Fair

$\square 5$ Poor

7. How would you rate your current mood?

(very unpleasant)

4

5

$5-6$

8. Do you engage in regular, physical activity?

$\square$ Yes

$\square$ No

If you answered "Yes," how many hours a week do you take part in physical activity? hours per week

9. If you engage in physical activity, would you consider your activity level to be:

$\square$ N/A

$\square$ Low (e.g., minimal walking)

$\square$ Moderate (e.g., brisk walking, yoga, moderate swimming, golfing, walking the dog)

$\square$ Vigorous (e.g., running, jogging, aerobics, swimming, biking)

10. Do you drink alcohol?

$\square$ Never

$\square$ Sometimes

$\square$ Often

$\square$ Very frequently

11. Do you have any vision problems (which are not fixed with glasses or contacts)?

$\square$ Yes (Please specify)

$\square$ No

12. Have you ever had a stroke? If yes, please specify how many years ago it occurred.

$\square$ Yes (Please specify) years ago

$\square$ No

13. Have you recently been feeling depressed?

$\square$ Yes

$\square$ No

14. Do you have difficulty hearing?

$\square$ Yes

$\square$ No (this includes having improved hearing from a hearing aid) 
Appendix (continued)

15. Are you a Native English speaker (fluent in English)? If no, how long have you been speaking English (years)? $\square$ Yes

$\square$ No (please specify how many years you have been speaking English) years

16. Do you speak more than one language?

$\square$ Yes

$\square$ No

If yes, how fluent are you in your other language(s):

Second language: 1

2 (not at all fluent)

Third language (if applies): 1 (not at all fluent)
2

3

4

4
trauma, and/or any other neurological conditions? If yes, please specify below.

$$
\begin{aligned}
& \square \text { Yes } \\
& \square \text { No }
\end{aligned}
$$

18. Do you have any issues sleeping?

$\square$ Yes

$\square$ No

On average, how many hours a night do you sleep?

Received July 31, 2015

Revision received October 30, 2015

Accepted November 17, 2015 